\title{
SMOKING CESSATION TREATMENTS: CURRENT PSYCHOLOGICAL AND PHARMACOLOGICAL OPTIONS
}

\author{
Leonor García-Gómez ${ }^{1}$, Andrea Hernández-PÉrez ${ }^{1 *}$, Valeri Noé-Díaz ${ }^{1}$, \\ JuAN ANTONIO RIEsCo-Miranda ${ }^{2,3}$ AND Carlos JIMÉNEZ-RUIZ ${ }^{4}$ \\ ${ }^{1}$ Department of Research in Tobacco and COPD, Instituto Nacional de Enfermedades Respiratorias Ismael Cosío \\ Villegas, Mexico City, Mexico; ${ }^{2}$ Department of Pulmonary Medicine, Hospital de San Pedro Alcántara, Cáceres, Spain; \\ ${ }^{3}$ Centro de Investigación Biomédica de Enfermedades Respiratorias, Spain and ${ }^{4}$ Smoking Cessation Service, Comunidad \\ Madrid, Spain
}

\begin{abstract}
Background: Smoking is considered an epidemic, indeed, one of the most important public health problems worldwide. It is also the most significant preventable cause of death, of a high number of premature deaths, and avoidable chronic diseases. It is considered an enormous economic burden for the world. Objective: To provide an overview of smoking-cessation treatments, including pharmacological and psychological options, and to gather current scientific evidence available on them. Methods: Research included reviewing publications from 2007-2018 in four databases using algorithms related to bupropion, varenicline, nicotine replacement therapy, smoking cessation, psychological treatment, motivational interview, cognitive-behavioral therapy and clinical guidelines for smoking treatment. Meta-analyses or systematic reviews and randomized or quasirandomized trials were selected. We also included clinical guidelines for smoking treatment from Mexico and other countries. Results: After refining the search, 37 articles met the criteria and were included in the review. The results were grouped by type of intervention. Conclusions: It is necessary to conduct research on combinations of both kinds of treatment with an integral, multidisciplinary vision. Current standard for smoking cessation is a combined psychological and pharmacological treatment. (REV INVEST CLIN. 2019;71:7-16)
\end{abstract}

Key words: Smoking cessation. Psychological treatment for smoking cessation. Pharmacological treatment for smoking cessation.

\section{INTRODUCTION}

Smoking is a chronic disorder that constitutes one of the most important public health problems worldwide. In fact, it is considered the leading preventable cause of death and the worst epidemic since the 20th

\section{Corresponding author:}

*Andrea Hernández-Pérez

Department of Research in Tobacco and COPD

Instituto Nacional de Enfermedades Respiratorias

Ismael Cosio Villegas (INER)

Calzada de Tlalpan, 4502

Col. Sección XVI, Del. Tlalpan

C.P. 14080, Mexico City, Mexico

E-mail: andrea.hernandez@iner.gob.mx century. Furthermore, the economic burden of smoking is considerable in many countries ${ }^{1,2}$. Since smoking cessation requires treating the addiction and modifying the smoker's behavior, psychological and pharmacological interventions are needed.
Received for publication: 08-06-2018

Approved for publication: 29-09-2018 doi: 10.24875/RIC.18002629 
The World Health Organization (WHO) estimates that there are over 1.1 billion smokers in the world ${ }^{1}$. $>7$ million deaths annually are related to the direct effects of tobacco, while some 890,000 results from exposure to secondhand smoke ${ }^{2}$. In Mexico, tobacco use is a health problem that frequently affects vulnerable groups such as children, adolescents, and women. At present, $17.6 \%$ of Mexicans over 15 years of age smoke tobacco, about 14.9 million people, while $14.9 \%$ of non-smokers are exposed to secondhand tobacco smoke in the home. The average age at which men and women begin to smoke is 19.3 years $^{3}$.

Besides nicotine, cigarettes contain over 4000 other substances, and carbon monoxide produced compromises oxygenation, just like any other biofuel when burned and inhaled ${ }^{4}$. These factors underlie the premature death rate of $50 \%$ among smokers and the estimated mean reduction of 10 years in life expectancy. Finally, the risk of cancer increases over 10-fold and smoking is associated with at least 25 other diseases ${ }^{5}$.

Therefore, the consequences of smoking have an enormous impact on public health-care costs. Indeed, reducing tobacco consumption is crucial to a population's overall health. Fortunately, 181 countries have signed the WHOs framework convention on tobacco control, which implements the strategies against tobacco use that is simplified in a policy package available to public health authorities worldwide ${ }^{1}$.

The purpose of this article is to provide an overview of psychological and pharmacological treatments for smoking cessation currently available.

\section{Search for information}

Research of publications on pharmacologic and psychological treatments was done separately. For pharmacologic approaches, our strategy included reviewing publications from 2007 to 2018 in two databases, Wiley-Blackwell Full Collection and EBSCOhost, using several algorithms: "bupropion," "varenicline," "nicotine replacement therapy (NRT)," and "smoking cessation." The searches generated 3522 and 135 hits, respectively. All articles were reviewed and those that met the following two criteria were included (1) meta-analyses or systematic reviews and (2) original articles that reported abstinence outcomes. Our review of published studies on psychological treatment for smoking cessation used the USAs National Center for Biotechnology Information (NCBI) database and The Cochrane Database of Systematic Reviews (CDSR) as search tools and the algorithms: "smoking cessation," "psychological treatment," "motivational interview," "cognitive-behavioral therapy," "behavioral therapy," and "clinical guidelines for smoking treatment." The publication period covered was also 2007-2018. The selection criteria were meta-analyses or systematic reviews, and randomized or quasi-randomized studies. This search identified 6038 hits in NCBI and 362 in CDSR, but only 23 articles satisfied our selection criteria.

The results of this research are presented by intervention type

\section{Brief advice to stop smoking}

All health professionals must be knowledgeable about the harmful effects of tobacco and the treatment options available to support cessation. To successfully approach smokers, health professionals must be able to speak convincingly and have excellent communication skills ${ }^{5}$. Patients should be asked if they smoke or live with smokers, accompanied by a thorough medical assessment (age at onset, use patterns, motivation to quit, and detecting obstacles to cessation), prescribing treatment, and doing follow-up until the goal of definitive cessation is reached ${ }^{6}$.

The term "brief advice" refers to a strategy that impacts people's motivation to quit smoking, seek treatment, or reduce their consumption of cigarettes through a single intervention. Compared to no intervention of any kind, brief advice has been shown to increase the frequency of quit attempts (risk ratio [RR] 1.24, 95\% confidence interval [Cl]: 1.16-1.33). Offering assistance in situ generates more quit attempts than giving advice based on medical grounds (RR 1.69, 95\% Cl: 1.24-2.31 for behavioral support and $1.39,95 \% \mathrm{Cl}: 1.25-1.54$ for offering medication) ${ }^{7}$.

Different models have been proposed to give brief advice on quitting. One of them is the $5 A^{\prime}$ s model, which includes the following steps: ask (about 
tobacco use); Advise (strongly suggest quitting); Assess (the subject's willingness to modify her/his behavior); Assist (set a date to begin the process); and Arrange (follow-up). Health professionals who lack the training required to prescribe drug treatment can turn to a model called AAC (Ask, Advise, and Connect $)^{4}$. This intervention does not demand much time or economic resources, so implementing it is recommended for all clinical contexts.

\section{Pharmacological treatment}

Clinical guidelines generally recommend dual treatment - medication plus counseling - because it doubles the probability of success. Some professionals do not recommend medication for smokers who consume fewer than 10 cigarettes a day, who some authors categorize as "light smokers." However, a smaller proportion of light smokers may also seek pharmacological support to quit. A study in the United Kingdom found that $18 \%$ of light smokers sought such support, mainly in the form of NRT and counseling. They also showed that $65 \%$ of light smokers had failed in their most recent attempt to quit. This suggests that even people who smoke only a few cigarettes a day often needs pharmacological or psychological help ${ }^{8}$. Smoking fewer than 10 cigarettes a day does not exempt people from the risk of acquiring any of the diseases associated with tobacco consumption, so they must also be strongly encouraged to quit ${ }^{5}$.

Pharmacological treatment for smoking cessation is designed to block the reinforcing effects of nicotine and reduce withdrawal symptoms. Treatments for smoking cessation can include NRT, bupropion, and varenicline ${ }^{6}$.

\section{NRT}

NRT involves administering gradually decreasing controlled doses of nicotine. It maintains relatively low plasma nicotine levels that ease anxiety and withdrawal symptoms by acting directly on the nicotinic cholinergic receptors $\alpha 4 \beta 29$. Various modalities exist transdermal patches, chewing gum, nasal spray, inhalers, and pills, but the availability of these products varies from country to country. Prescribing elevated doses of nicotine coupled with prolonged treatment
(>14 weeks) may be a useful strategy in smokers with high dependence. Some patients who use the transdermal patch experience insomnia, but the patch can be removed at night without reducing effectiveness, since using it during the day is as efficacious as using it for 24 h. ${ }^{5}$ NRT does not increase the risk of adverse cardiovascular effects in smokers with a history of cardiovascular disease ${ }^{10}$, although the exact therapeutic dose must be determined based on each patient's needs and tolerance, as well as the issues of cost and accessibility ${ }^{11}$.

\section{Bupropion}

Bupropion was the first non-nicotinic drug treatment approved by the FDA to stop smoking. An antidepressant that stimulates noradrenergic and dopaminergic functions, it has been shown to double abstinence rates even in the long term, which is why it is used as a first-line drug to treat smoking ${ }^{6}$. It is not, however, indicated in smokers with a history or risk of seizures, or in pregnant or breastfeeding women ${ }^{5}$.

Bupropion's effectiveness has been reported in populations with psychiatric disorders such as schizophrenia, depression, and post-traumatic stress disorder, while some observations suggest that it prevents the weight gain commonly associated with abstinence and restores sexual functioning ${ }^{12}$.

Bupropion can also be indicated in lung cancer patients undergoing surgery and chemotherapy as a means of controlling affective symptoms associated with the withdrawal syndrome. However, monitoring is recommended, since the drug can decrease a person's normal appetite, which is not desirable in patients under cancer treatment ${ }^{13,14}$.

\section{Nicotine receptor partial agonists}

Varenicline is a partial agonist of nicotinic $\alpha 4 \beta 2$ cholinergic receptors that exhibit 20 times greater affinity for these receptors than nicotine. On binding to those receptors, varenicline blocks their response and promotes the release of dopamine into the mesolimbic system, on which the positive reinforcement of smoking behavior depends on. Thus, it blocks the reinforcing effect of nicotine. In addition, it has been reported that varenicline has a nicotinic receptor 
blocking effect due to its role as a partial agonist. In this way, varenicline helps decrease the risk of relapse in smokers by producing anhedonia in relation to smoking ${ }^{15}$. These effects reduce craving and moderate withdrawal syndrome ${ }^{16}$. A dose of $1 \mathrm{mg} /$ day is effective and doubles the chances of quitting, while 2 $\mathrm{mg} /$ day increases the likelihood of long-term abstinence threefold. This drug is well tolerated for up to 6 months and in prolonged treatments of up to 1 year reduces the likelihood of relapse ${ }^{5}$. Varenicline was approved by the FDA in 2006 and is now recommended for smoking cessation by several international guidelines for clinical practice ${ }^{17}$.

Varenicline does not seem to be associated with an increased risk of depression or self-injurious behavior, compared to NRT ${ }^{18}$. In fact, recent evidence suggests that it can be prescribed widely, even to disease-specific groups of patients (i.e., cardiovascular, chronic obstructive pulmonary disease, schizophrenia, and other psychiatric disorders, depression) to help more smokers achieve abstinence ${ }^{17}$. However, careful checkup and follow-up are recommended ${ }^{19}$.

The EAGLES study evaluated neuropsychiatric safety and efficacy of varenicline, bupropion, and nicotine patches in smokers with and without psychiatric disorders. It is widely recognized for its rigorous analysis of scientific evidence. It underscores that:

a. In smokers with no history of psychiatric pathology, varenicline, bupropion, and nicotine patches have been shown to be more effective in achieving cessation than a placebo. Varenicline odds ratio (OR): 2.99 (2.33, 3.83); bupropion OR: 2.00 (1.54, 2.59); and nicotine patches OR; 1.96 (1.51, 2.54), at 6 months of follow-up. Varenicline was more effective than bupropion and nicotine patches, with OR: 1.96 (1.51, 2.54) and 1.52 (1.23, 1.89), respectively. No significant differences were found between bupropion and the nicotine patches.

b. In the group of smokers with controlled neuropsychiatric disease or a history of such disease, a study data showed that varenicline, bupropion, and nicotine patches were all more effective in helping subjects quit smoking than a placebo. Varenicline OR: 2.50 (1.90, 3.29); bupropion OR: 1.77 (1.33, 2.36); and nicotine patches OR: 1.65 (1.24, 2.20), at 6 months of follow-up. Varenicline was more effective than bupropion and nicotine patches, with OR: $1.41(1.11,1.79)$ and $1.51(1.19,1.93)$, respectively. No significant differences were found between bupropion and the nicotine patches.

c. None of these treatments - varenicline, bupropion, or nicotine patches - increased the incidence of adverse neuropsychiatric effects in smokers without disorders of this nature, or in smokers with controlled neuropsychiatric disease or a history of neuropsychiatric disease. In this regard, the EAGLES study showed that using varenicline, bupropion, or nicotine patches did not increase the occurrence of suicidal ideation or behavior in the group of smokers without neuropsychiatric disorders or in smokers with neuropathy, controlled neuropsychiatric disease, or a history of neuropsychiatric disease.

Today, more than ever, we know that these medications designed to stop smoking are effective and safe.

Cytisine has a molecular structure similar to varenicline but is only available in countries in Central and Eastern Europe. It is a good option for smokers who want to quit and live in socially or economically depressed areas because it is more affordable. Recent studies have confirmed its effectiveness, although the long-term cessation rates reported are only modest ${ }^{17}$. Several clinical trials have shown its efficacy, but this drug has not yet been approved by the FDA or any other health organization in the Western hemisphere $^{13}$.

\section{Vaccines}

Vaccine development is another area of exploration. The primary mechanism of a vaccine would consist in generating antibodies that bind to nicotine to reduce its entry into the brain. A vaccine, however, would need to be compatible and complementary with other smoking-cessation treatments. It would be indicated for the following groups of subjects: (a) heavy smokers who have made multiple unsuccessful attempts to quit using different methods; (b) patients who are highly motivated to quit; and (c) patients who have positive attitudes toward vaccines ${ }^{20}$. Modifications designed to improve serum antibody affinity are being tested and may improve the performance of future clinical candidate vaccines ${ }^{21-23}$. 
Table 1. Odds ratios and risk ratios of pharmacological and behavioral interventions for smoking abstinence of 6 months or more.

\begin{tabular}{|c|c|c|c|}
\hline Study & $\mathrm{n}$ & Treatment & Odds ratio-risk ratio \\
\hline \multirow[t]{3}{*}{ Kruger et al. ${ }^{43}$} & \multirow[t]{3}{*}{10,801 subjects } & Brief advice (5A's model) & $\mathrm{OR}=11.2,95 \%, 7.1-17.5$ \\
\hline & & Medication & $\mathrm{OR}=6.2,95 \%, 4.3-9$ \\
\hline & & $\begin{array}{l}\text { Brief advice ( } 5 A^{\prime} \text { 's model) + } \\
\text { Medication }\end{array}$ & $\mathrm{OR}=6.2,95 \%, 9.3-2.3$ \\
\hline \multirow[t]{6}{*}{ Cahill et al. ${ }^{11}$} & \multirow[t]{6}{*}{14 trials } & NRT & $\mathrm{OR}=1.84,95 \%, 1.71-1.99$ \\
\hline & & Bupropion & $\mathrm{OR}=1.82,95 \%, 1.60-2.06$ \\
\hline & & Varenicline & $\mathrm{OR}=2.88,95 \%, 2.40-3.47$ \\
\hline & & Bupropion versus NRT & $\mathrm{OR}=0.99,95 \%, 0.86-1.13$ \\
\hline & & Varenicline versus NRT & $\mathrm{OR}=1.57,95 \%, 1.29-1.91$ \\
\hline & & Varenicline versus bupropion & $\mathrm{OR}=1.59,95 \%, 1.29-1.96$ \\
\hline \multirow[t]{3}{*}{ Stead et al. ${ }^{25}$} & $\begin{array}{l}66 \text { trials } \\
\mathrm{n}=4395\end{array}$ & $\begin{array}{l}\text { Group program versus self-help } \\
\text { program }\end{array}$ & $R R=1.88,95 \%, 1.52-2.33$ \\
\hline & $\begin{array}{l}14 \text { trials } \\
\mathrm{n}=7286\end{array}$ & Group program versus brief support & $\mathrm{RR}=1.22,95 \%, 1.03-1.43$ \\
\hline & $\begin{array}{l}\text { Nine trials } \\
\mathrm{n}=1098\end{array}$ & $\begin{array}{l}\text { Group program compared to no } \\
\text { intervention controls }\end{array}$ & $R R=2.60,95 \%, 1.80-3.76$ \\
\hline Stead et al. ${ }^{44}$ & $\begin{array}{l}52 \text { randomized or quasi- } \\
\text { randomized controlled trials } \\
\mathrm{n}=19,488 \text { participants }\end{array}$ & $\begin{array}{l}\text { Combination pharmacotherapy and } \\
\text { behavioral treatment versus usual } \\
\text { care or brief advice or less } \\
\text { intensive behavioral support }\end{array}$ & $R R=1.83,95 \%, 1.68-1.98$ \\
\hline $\begin{array}{l}\text { Lindson-Hawley } \\
\text { et al. }{ }^{31}\end{array}$ & $\begin{array}{l}28 \text { randomized controlled trials } \\
\mathrm{n}=16,803 \text { participants }\end{array}$ & $\begin{array}{l}\text { Motivational interviewing versus } \\
\text { brief advice or usual care }\end{array}$ & $R R=1.26,95 \%, 1.16-1.36$ \\
\hline Hiscock et al. ${ }^{30}$ & $\begin{array}{l}202,084 \text { records of smokers } \\
\text { in England who attended } \\
\text { an NHS stop smoking service }\end{array}$ & $\begin{array}{l}\text { One-to-one counseling versus open } \\
\text { group forms of behavioral therapy }\end{array}$ & $\mathrm{OR}=1.26,95 \%, 1.12-1.41$ \\
\hline $\begin{array}{l}\text { Baskerville } \\
\text { et al. }{ }^{33}\end{array}$ & $\begin{array}{l}\mathrm{n}=238 \text { from } 19 \text { to } 29 \text {-year-old } \\
\text { smokers }\end{array}$ & $\begin{array}{l}\text { Multicomponent web-based and } \\
\text { social media approach known as } \\
\text { BIO }(n=102) \text { versus group of } \\
\text { smokers' helpline }(n=136)\end{array}$ & $\mathrm{OR}=2.95,95 \%, 1.56-5.57$ \\
\hline
\end{tabular}

NRT: nicotine replacement therapy, BIO: break-it-off.

Recently, advances have been published on the elaboration of lipid-polymeric hybrid nanoparticle (NP)-based nicotine nanovaccines. Nicotine hapten is such a small molecule that it only elicits an immune response when attached to a carrier, such as a protein or NP. Zhao et al. designed a series of nanovaccines, and their studies with mice showed that certain types of NPs exhibited the greatest ability to block nicotine from entering the brain ${ }^{24}$. Unfortunately, the assays conducted to date have revealed drawbacks that prevent these systems from achieving higher cessation rates than a placebo ${ }^{20}$.

\section{Combined treatment with medications}

Numerous trials have demonstrated that combined interventions increase the probability of successful outcomes (Table 1). These may involve complementing specialized psychological intervention with medication or combined pharmacological treatment. For instance, combined NRT has been associated with higher abstinence rates than single NRT formulations, while varenicline combined with NRT has been associated with similar smoking-cessation rates (OR, 1.06; $95 \%$ credible interval [Crl], 0.75-1.48), and both were 
associated with higher rates of long-term abstinence compared to bupropion or single formulations of NRT (OR, 1.06; 95\% Crl, 0.75-1.48) $)^{11}$.

\section{Psychological treatment}

Among the non-pharmacological therapeutic options available for smoking cessation, psychological treatments stand out. They can include brief counseling, individual or group cognitive-behavioral therapy, telephone counseling, and the use of information technologies such as mobile applications or internet pages with digital programs designed to help achieve cessation. The scientific literature reports that individual behavioral interventions are more effective than brief advice, self-help materials, or counseling.

A review of 52 studies (with 19,488 participants) provided high-quality evidence for the benefits of combined pharmacotherapy plus behavioral treatment compared to usual care, brief advice, or less intensive behavioral support (RR $1.83,95 \% \mathrm{Cl} 1.68$ $1.98)^{25-27 .}$

Behavioral therapies were among the first psychological treatments that proved effective in smoking cessation. They began with aversive-type approaches (e.g., fast smoking) and then were complemented with self-observation, relaxation exercises, stimulus control, and gradual reduction of nicotine and tar intake, among other measures. Later, multicomponent psychological treatments and relapse prevention strategies appeared ${ }^{28}$. Behavioral therapies emphasize the social and contextual aspects of smoking and have been shown to enhance overall success rates. The objectives of this study are as follows: (1) to lead them to abstinence and (2) to learn techniques to prevent smoking in stimulating contexts $^{29}$. Therapy pursues short-term and specific goals, focusing on current situations instead of past ones, and seeking to identify diverse solutions rather than causes ${ }^{30}$.

The stages of change model by Prochaska and DiClemente evaluate a patient's readiness for change and propose that abstinence should not be considered as a specific event in the smoker's life, but as a process made up of various stages. It proposes six basic stages: precontemplation, preparation for action, action, maintenance, and, in some cases, relapse. To determine at what stage the patient is may be useful to generate an intervention aimed to specific needs ${ }^{5}$.

Brief motivational interventions and motivational interviewing strategies focus on exploring smokers' feelings, beliefs, ideas, and values regarding tobacco use in an effort to uncover any ambivalence toward $i^{6}$. The four guiding principles of motivational interview techniques are as follows: (a) expressing empathy; (b) developing discrepancy; (c) rolling with resistance; and (d) supporting self-efficacy.

In systematic reviews, motivational interview is usually compared to (1) self-help smoking-cessation material; (2) in-person/telephone-based smoking-cessation support; (3) in-person smoking health warnings; and (4) no smoking-cessation intervention. When compared to self-help materials or no smoking-cessation intervention, the effect of the motivational interview was found to be non-significant, as the pooled self-help control studies had an RR of 1.11 (0.91-1.35; 6 trials, $n=3502 ; I^{2}=0 \%$ ), while the pooled no smoking-cessation control RR was 0.85 (0.61-1.19; 2 trials, $n=755 ; I^{2}=0 \%$ ). However, when compared to a control of in-person/telephone smoking-cessation support or an in-person smoking health warning, the motivational interview for smoking cessation has shown significant benefits (RR $1.31 ; 95 \% \mathrm{Cl} 1.19$ $1.45 ; 17$ trials, $n=10,966 ; I^{2}=54 \%$ and RR 2.25; $95 \% \mathrm{Cl} 1.41-3.57 ; 2$ trials, $n=945 ; \mathrm{I}^{2}=0 \%$, respectively) $)^{31}$.

Fiore et al. reported the effects of various interventions, indicating ORs of 1.7 for individual behavioral counseling and 1.3 for group behavioral counseling ${ }^{6}$. In addition, the longer the contact time (OR $=3.2$ for $91-300 \mathrm{~min}$ ) and the greater the number of sessions $(\mathrm{OR}=1.9$ for $4-8$ sessions, and $\mathrm{OR}=$ 2.3 for $>8$ sessions), the more effective the treatment will be. Of the existing psychological techniques, the following have been shown to have ORs above 1 : gradual reduction of cigarettes $(O R=1.1$ ); management of negative affect $(O R=1.2)$; social support during treatment $(O R=1.3)$; social support outside of the treatment $(O R=1.5)$; training in problem-solving $(O R=1.5)$; other aversive procedures $(O R=1.7)$; and the rapid-smoking technique $(\mathrm{OR}=2.0)^{6}$. 
Another systematic review of effective psychological treatments for smoking cessation with RRs of 1.301.98 mentions the following: group therapy ( $R R=$ $1.98)$; individual therapy $(R R=1.36)$; and self-help procedures $(R R=1.45)$, among others.

New technologies and social networks have been incorporated into smoking-cessation interventions. A study has reported that a digital social media platform can effectively complement traditional cessation services for young adult smokers seeking help to quit ${ }^{32,33}$.

Recently, researchers have turned to the study of so-called third-generation psychological treatments such as behavioral activation and mindfulness, and some clinical trials have proven their effectiveness. A randomized controlled trial conducted with a pilot parallel group based on a brief mindfulness practice intervention delivered to smokers by a personal digital assistant revealed that when compared to sham meditation, the brief mindfulness approach reduced the overall negative affect: $F(1,1798)=13.8, p=0.0002$. Craving diminished immediately post-meditation (Group $\times$ Assessment interaction type: $F(2,1796)=12.3$, $p=0.0001$ ), and the number of cigarettes smoked per day decreased over time (Group $\times$ Day interaction, $F(1,436)=5.50, p=0.01)^{34}$.

Treatment should be personalized, especially in the case of consumers of other psychoactive substances and/or smokers with a history of depression because psychiatric symptoms are related to a greater probability of relapse 27,35 .

\section{Relapse in smoking-cessation treatment}

Even though a smoking-cessation treatment may seem to be effective during the first 4 weeks, relapse rates increase with time. Follow-up in several studies reports abstinence rates of around $40 \%$ at 12 months after finishing treatment. One of the strategies that have been explored to improve abstinence rates consists in adding regular exercise to usual pharmacological treatment. Results, however, have been disappointing, as there are no reports that regular exercise has a significant effect on abstinence rates at 12 months ( 23 vs. $21.2 \%)^{36}$.
Extended pharmacological treatment may be an option that raises post-treatment abstinence duration, especially in smokers who find it difficult to begin abstinence ${ }^{37}$. In a randomized controlled trial in which participants successfully reached abstinence for 7 days, varenicline use continued for up to 52 weeks in a non-treatment follow-up phase. Abstinence rates were significantly higher for participants who received varenicline than for those who were given a placebo (43.6 vs. $36.9 \%)^{38}$. Despite all these efforts, however, the solution to maintaining longterm abstinence remains beyond our reach. Therefore, this is a field of research in which we must continue our search to find effective strategies.

\section{Implementation of tobacco cessation}

Some smokers who want to quit may be able to do so with minimal measures such as self-advice, brief advice, or techniques that promote ambivalence regarding tobacco consumption. Additional help can be offered by nicotine substitutes and more formal psychological therapies. The use of other medications, meanwhile, might be best reserved for specialized care. Formal clinics offering multidisciplinary treatment methods are required for the severely addicted smokers or those with comorbidities with other addictions or psychological disorders. From a public health perspective, nicotine patches should be included on the list of essential drugs or medications available free of charge through public health systems since this would promote and facilitate their use and increase abstinence and maintenance rates.

Recommendations gathered from various clinical guidelines have been formulated in a pyramidal health model, in which each level represents a specific sector of the health-care system, and the actions that need to be carried out there to achieve optimal attention to smoking and comply with the conventions and laws established for tobacco control.

Recommendations for health care workers are that all of them should take every opportunity to offer brief advice on the advantages of quitting to all their patients who smoke. The first level of medical care is an ideal place to identify and treat tobacco users, at least, with brief interventions as part of routine services. Indeed, primary care providers can help patients successfully quit smoking by offering interventions as short as 
Figure 1. Pyramid model of smoking cessation at different levels of care.

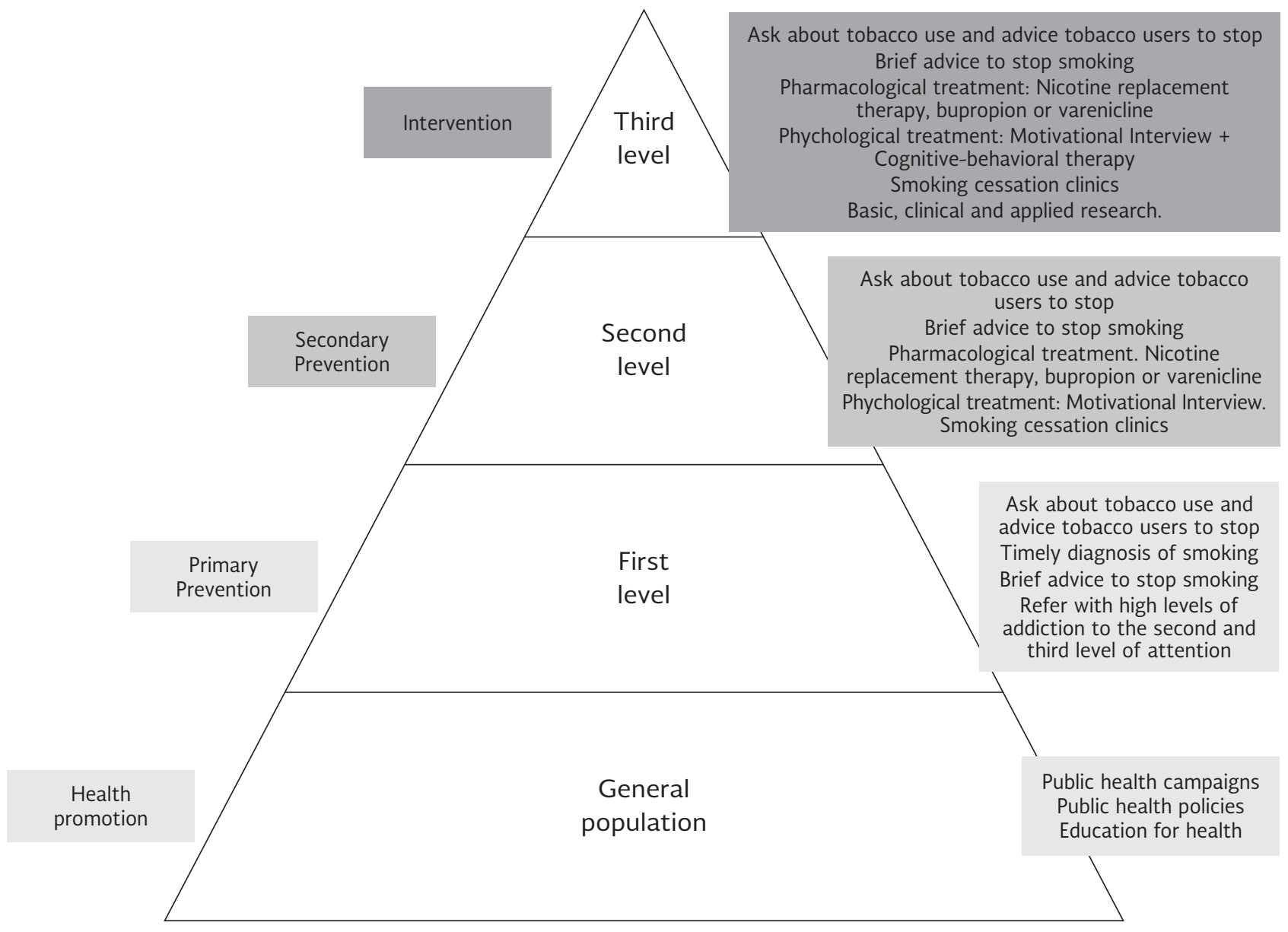

3 min. Unfortunately, $<50 \%$ of primary care providers worldwide routinely ask and/or advise patients to quit smoking. Recommendations in clinical guidelines focus on secondary and tertiary prevention approaches that involve medical and psychological support for smokers and specialized treatment that includes brief advice, psychological treatment (cognitive-behavioral), NRT, or first-line medications (bupropion and varenicline) to reduce the health risks related to smoking, improve the prognosis if a disease already exists, and enhance patients' quality of life (Fig. 1) 6,39-42.

\section{CONCLUSIONS}

Evidence has demonstrated consistently that combining pharmacological treatment with behavioral intervention doubles the probability of success of the smoking cessation. Clinical guidelines in several countries recommend cognitive-behavioral intervention together with the administration of the first-line drugs, that is, NRT, bupropion, or varenicline. It has also been recognized that brief advice for smoking cessation should be given in all health-care contexts. In the first- and second-level health-care facilities, most professionals ask about tobacco use but rarely discuss pharmacological or psychological interventions. To achieve integration of smoking assessment and intervention at all levels of health care, trained personnel and public health policies are needed to design and structure the systematic monitoring of compliance with antismoking measures.

The global prevalence of smoking has not decreased significantly in the past 10 years. One reason for this may be that health systems do not identify and register tobacco consumption beginning at the first level of care. 
The $\mathrm{WHO}$ and other international organizations promote action programs that include schemes for cessation at different levels of clinical care. However, these programs are not always reproduced accurately at the local level. Sometimes, uncoordinated actions are performed, in which only specialized thirdlevel care clinics are created, but training of professionals in the first and second levels of care is not reinforced. Without doubt, health professionals play a fundamental role in giving attention to smoking, so even a minimal intervention on their part could bring benefits for the general population in terms of promoting abstinence and establishing smoke-free spaces.

Finally, current options for smoking cessation - both pharmacological and psychological - have proven to be quite effective in the short term. However, in the following year, abstinence rates are disappointing. New options have been explored; for example, freeing up the market for cytisine could spur smoking cessation, especially in developing countries. With respect to psychosocial and behavioral interventions, a wave of new therapies - known as "third generation" - has emerged and has shown significant results on abstinence in clinical trials. Nevertheless, evidence of their effectiveness on maintaining abstinence is needed. Another significant area of research is working with novel technologies that use the internet and mobile devices as important tools that complement psychological treatment since they can be used to deliver information on cessation to broad audiences, to focus and intensify counseling for those who are attempting to quit, and to foster social support, which is invaluable in the cessation process.

\section{REFERENCES}

1. World Health Organization (WHO). WHO Report on the Global Tobacco Epidemic, 2017. World Health Organization. 2017. p. 1-263. [Last accessed: Jan 5, 2018]

2. Mackay J, Schluger N. Global tobacco epidemic. Tob Epidemic. 2015;42:19-26. [Last accessed: Dec 20, 2017].

3. Reynales-Shigematsu LM, Zavala-Arciniega L, Paz-Ballesteros WC, et al. Encuesta Nacional de Consumo de Drogas, Alcohol y Tabaco, Mexico 2016-2017; 2017

4. Sansores M, Regalado P, Giraldo B, et al. Guías mexicanas para el tratamiento del tabaquismo. Un consenso nacional de expertos. Neumol Cir Torax. 2005;64:S84-112.

5. de Salud S. Prevención, Diagnostico y Tratamiento del Consumo y Humo Ajeno en el Primer Nivel de Atención. 2009. [Last accessed: Mar 8, 2018].

6. Fiore MC, Jaen CR, Baker T, et al. Treating Tobacco Use and Dependence: 2008 Update. [Last accessed: Feb 21,2018]. USA DHHS; 2008.
7. Aveyard P, Begh R, Parsons A, West R. Brief opportunistic smoking cessation interventions: a systematic review and meta-analysis to compare advice to quit and offer of assistance. Addiction. 2012;107:1066-73.

8. Kotz D, Fidler J, West R. Very low rate and light smokers: smoking patterns and cessation-related behaviour in england, 200611. Addiction. 2012;107:995-1002.

9. Jiloha RC. Biological basis of tobacco addiction: implications for smoking-cessation treatment. Indian J Psychiatry. 2010;52:301-7.

10. Stead LF, Perera R, Bullen C, et al. Nicotine replacement therapy for smoking cessation. Cochrane Database Syst Rev. 2012; 11:CD000146.

11. Cahill K, Stevens S, Lancaster T. Pharmacological treatments for smoking cessation. JAMA. 2014;311:193-4.

12. Roberts E, Eden Evins A, McNeill A, Robson D. Efficacy and tolerability of pharmacotherapy for smoking cessation in adults with serious mental illness: a systematic review and network metaanalysis. Addiction. 2016;111:599-612.

13. Karam-Hage M, Cinciripini PM, Gritz ER. Tobacco use and cessation for cancer survivors: an overview for clinicians. CA Cancer J Clin. 2014;64:272-90.

14. Leone FT, Evers-Casey S, Toll BA, Vachani A. Treatment of tobacco use in lung cancer: diagnosis and management of lung cancer, 3rd ed: American college of chest physicians evidencebased clinical practice guidelines. Chest. 2013;143:e61S-e77S.

15. Fagerström K, Hughes J. Varenicline in the treatment of tobacco dependence. Neuropsychiatr Dis Treat. 2008;4:353-63.

16. Muramoto ML, Leischow SJ, Sherrill D, Matthews E, Strayer LJ. Randomized, double-blind, placebo-controlled trial of 2 dosages of sustained-release bupropion for adolescent smoking cessation. Arch Pediatr Adolesc Med. 2007;161:1068-74.

17. Cahill K, Lindson-Hawley N, Thomas K, Fanshawe T, Lancaster T. Nicotine Receptor Partial Agonists for Smoking Cessation. [Last accessed: February 2, 2018]. Summary of Findings for the Main Comparison. The Cochrane Library; 2016.

18. Kotz D, Viechtbauer W, Simpson C et al. Cardiovascular and neuropsychiatric risks of varenicline: a retrospective cohort study. Lancet Respir Med. 2015;3:761-8.

19. Baker TB, Piper ME, Stein JH, et al. Effects of nicotine patch vs varenicline vs combination nicotine replacement therapy on smoking cessation at 26 weeks: a Randomized clinical trial. JAMA. 2016;315:371-9.

20. Garrido P, Prat A, Crespo I, Valverde A, Salvador E. Vacunas para la deshabituación tabáquica. Vacunas. 2012;13:163-70.

21. Zhao Z, Hu Y, Harmon T, et al. Rationalization of a nanoparticlebased nicotine nanovaccine as an effective next-generation nicotine vaccine: a focus on hapten localization. Biomaterials. 2017;138:46-56

22. Zeigler DF, Roque R, Clegg $\mathrm{CH}$. Construction of an enantiopure bivalent nicotine vaccine using synthetic peptides. PLoS One. 2017;12:e0178835.

23. Thorn JM, Bhattacharya K, Crutcher R, et al. The effect of physicochemical modification on the function of antibodies induced by anti-nicotine vaccine in mice. Vaccines (Basel). 2017;5:e11.

24. Zhao Z, Harris B, Hu Y, et al. Rational incorporation of molecular adjuvants into a hybrid nanoparticle-based nicotine vaccine for immunotherapy against nicotine addiction. Biomaterials. 2018; 155:165-75

25. Stead LF, Carroll AJ, Lancaster T. Group behaviour therapy programmes for smoking cessation. Cochrane Database Syst Rev. 2017;3:CD001007.

26. Lancaster T, Stead LF. Individual behavioural counselling for smoking cessation. Cochrane Database Syst Rev. 2017;3: CD001292.

27. Stead LF, Koilpillai $P$, Lancaster T. Additional behavioural support as an adjunct to pharmacotherapy for smoking cessation. Cochrane Database Syst Rev. 2015;10:CD009670.

28. Hilbert A, Buerger A, Hartmann AS, et al. Psychometric evaluation of the eating disorder examination adapted for children. Eur Eat Disord Rev. 2013;21:330-9.

29. George T. Nicotina y tabaco. In: Tratado de Medicina Interna. [Last accessed: Jan 3,2018]. 2017. p. 9113.

30. Hiscock R, Murray S, Brose LS, et al. Behavioural therapy for smoking cessation: the effectiveness of different intervention types for disadvantaged and affluent smokers. Addict Behav. 2013;38:2787-96.

31. Lindson-Hawley N, Thompson TP, Begh R. Motivational interviewing for smoking cessation. Cochrane Database Syst Rev. 2015;3:CD006936.

32. Ramo DE, Thrul J, Delucchi KL, et al. The tobacco status project (TSP): study protocol for a randomized controlled trial of a facebook smoking cessation intervention for young adults. BMC Public Health. 2015;15:897. 
33. Baskerville NB, Azagba S, Norman C, McKeown K, Brown KS Effect of a digital social media campaign on young adult smoking cessation. Nicotine Tob Res. 2016;18:351-60.

34. Ruscio AC, Muench C, Brede E, Waters AJ. Effect of brief mindfulness practice on self-reported affect, craving, and smoking: a Pilot randomized controlled trial using ecological momentary assessment. Nicotine Tob Res. 2016;18:64-73.

35. Joly B, Perriot J, d'Athis $P$, et al. Success rates in smoking cessation: psychological preparation plays a critical role and interacts with other factors such as psychoactive substances. PLoS One. 2017;12:e0184800.

36. Abrantes AM, Strong DR, Lloyd-Richardson EE, et al. Regular exercise as a protective factor in relapse following smoking cessation treatment. Am J Addict. 2009;18:100-1.

37. Jiménez-Ruiz CA, Fagerström KO. Smoking cessation treatment for COPD smokers: the role of pharmacological interventions. Monaldi Arch Chest Dis. 2013;79:27-32.

38. Tonstad S, Tønnesen P, Hajek $P$, et al. Effect of maintenance therapy with varenicline on smoking cessation: a randomized controlled trial. JAMA. 2006;296:64-71.
39. Turk ML, El-Khoury JR. Additional research is needed to improve smoking cessation in developing countries. Addiction. 2014;109:510.

40. Training NC for SC and Local Stop Smoking Services. Service and Delivery Guidance 2014; 2014.

41. Bissell K. Smoking Cessation and Smoke-Free Environments for Tuberculosis Patients. International Union Against Tuberculosis and Lung Disease; 2010.

42. Ministry of Health. New Zealand Guidelines for Helping People to Stop Smoking. [Last accessed: Nov 22, 2017]. 2014 p. 1-9.

43. Kruger J, O'Halloran A, Rosenthal AC, Babb SD, Fiore MC. Receipt of evidence-based brief cessation interventions by health professionals and use of cessation assisted treatments among current adult cigarette-only smokers: national adult tobacco survey, 2009-2010. BMC Public Health. 2016; 16:141.

44. Stead LF, Koilpillai P, Fanshawe TR, Lancaster T. Combined pharmacotherapy and behavioural interventions for smoking cessation. Cochrane Database Syst. Rev 2016;3:CD008286. 